\title{
STRUCTURAL STABILITY OF VECTOR FIELDS WITH ORBITAL INVERSE SHADOWING
}

\author{
Keonhee Lee, Zoonhee Lee, And Yong Zhang
}

\begin{abstract}
In this paper, we give a characterization of the structurally stable vector fields via the notion of orbital inverse shadowing. More precisely, it is proved that the $C^{1}$ interior of the set of $C^{1}$ vector fields with the orbital inverse shadowing property coincides with the set of structurally stable vector fields. This fact improves the main result obtained by K. Moriyasu et al. in [15].
\end{abstract}

\section{Introduction}

Structurally stable systems (both diffeomorphisms and flows) were the main objects of interest in the global qualitative theory of dynamical systems in the last 40 years. Now we know that structural stability for flows is equivalent to Axiom A combined with the strong transversality condition (see $[6,25]$ ).

One of the most important properties of a structurally stable system is the shadowing property (also known as the pseudo orbit tracing property). The shadowing property is the key of the analysis of such diffeomorphisms or flows.

A long time ago, various approaches were applied to show that a structurally stable diffeomorphism has the shadowing property. But the fact that a structurally stable flow has the shadowing property was proved by S. Pilyugin recently (see [17]).

The main difficulty of the shadowing problem for a structurally stable flow is created by the following fact specific for flows. Let $p$ be a nonwandering point of a structurally stable flow. Then the trajectory of $p$ is hyperbolic. Denote by $S(p)$ and $U(p)$ the corresponding "stable" and "unstable" subspaces of the hyperbolic structure. If $p_{1}$ is a rest point, and $p_{2}$ belongs to a nonsingular nonwandering trajectory, then

$$
\operatorname{dim}\left(S\left(p_{1}\right)+U\left(p_{1}\right)\right) \neq \operatorname{dim}\left(S\left(p_{2}\right)+U\left(p_{2}\right)\right) .
$$

Received January 21, 2006; Revised October 25, 2007.

2000 Mathematics Subject Classification. Primary 37C; Secondary 37D.

Key words and phrases. flow, method, orbital inverse shadowing, shadowing, structurally stable, vector field.

This work was supported by the KRF Grant funded by the Korean Government (MOEHRD) (KRF-2005-070-C00015). 
Hence the hyperbolic structures near rest points and near nonsingular nonwandering trajectories are qualitatively different. Consequently the standard shadowing approaches do not work. In fact, it was proved by C. Robinson [22] and K. Sakai [23] that the $C^{1}$ interior of the set of diffeomorphisms with the shadowing property coincides with the set of structurally stable diffeomorphisms. However it is still open problem whether the above results can be applied to the case of flows; i.e., is a flow in the $C^{1}$ interior of the set of flows (or vector fields) with the shadowing property structurally stable?

As a partial answer of the above problem, very recently, K. Lee and K. Sakai [13] proved that every nonsingular flow (or vector field) in the $C^{1}$ interior of the set of flows (or vector fields) with the shadowing property is structurally stable. To prove this, they used the fact that every nonsingular star flow satisfies Axiom A and the no-cycle condition which is proved by S. Gan and L. Wen [4]. Unfortunately, a star flow with singularities may not satisfy Axiom A as you can see in [4].

We are going to prove that a flow (with singularities) in the $C^{1}$ interior of the set of flows with the inverse shadowing property which is a "dual" notion of shadowing property is structurally stable.

The concept of inverse shadowing for homeomorphisms "dual" to the shadowing was established by R. Corless and S. Pilyugin [2], and P. Kloeden et al. $[7,8]$ redefined this property using the concept of a method. Generally speaking, a homeomorphism has the inverse shadowing property with respect to a class of methods if any trajectory can be uniformly approximated with given accuracy by a $\delta$-pseudotrajectory generated by a method from the chosen class if $\delta>0$ is sufficiently small. An appropriate choice of the class of admissible pseudotrajectories is crucial here (see $[3,9,18]$ ).

It was shown by S. Pilyugin [18] that every structurally stable diffeomorphism has the inverse shadowing property with respect to the class of continuous methods. Recently, K. Lee and Z. Lee [10] introduced the notion of inverse shadowing for flows and showed that every expansive flow with the shadowing property has the inverse shadowing property with respect to the class of continuous methods. Moreover, Y. Han and K. Lee [5] proved that every structurally stable flow has the inverse shadowing property with respect to the class of continuous methods. Moreover, S. Pilyugin et al. [19] proved that the $C^{1}$ interior of the set of diffeomorphisms having the orbital shadowing property coincides with the set of structurally stable diffeomorphisms.

In this paper, we introduce the notion of orbital inverse shadowing for vector fields and prove that the $C^{1}$ interior of the set of vector fields with the orbital inverse shadowing property (or inverse shadowing property) coincides with the set of structurally stable vector fields. This fact improves the main result obtained by K. Moriyasu et al. in [15]. 


\section{Preliminaries}

Let $M$ be a $C^{\infty}$ closed manifold and let $d$ be the distance on $M$ induced by a Riemannian metric $\|\cdot\|$ on the tangent bundle $T M$. Denote by $\mathcal{X}^{1}(M)$ the set of all $C^{1}$ vector fields on $M$ endowed with the $C^{1}$ topology generated by the $C^{1}$ metric $d_{C^{1}}$. Every $X \in \mathcal{X}^{1}(M)$ generates a $C^{1}$ flow $X_{t}: \mathbb{R} \times M \longrightarrow M$; that is a $C^{1}$ map such that $X_{t}: M \longrightarrow M$ is a diffeomorphism satisfying $X_{0}(x)=x$ and $X_{t+s}(x)=X_{t}\left(X_{s}(x)\right)$ for all $s, t \in \mathbb{R}$ and $x \in M$.

Let $\delta>0$ and $T>0$ be arbitrary. We say that a mapping $\phi: \mathbb{R} \longrightarrow M$ is a $(\delta, T)$-pseudotrajectory of $X \in \mathcal{X}^{1}(M)$ if, for any $t \in \mathbb{R}$,

$$
d\left(X_{s}(\phi(t)), \phi(s+t)\right)<\delta
$$

for $|s| \leq T$. A mapping $\Psi: \mathbb{R} \times M \longrightarrow M$ is called a $(\delta, T)$-method for $X$ if, for any $x \in M$, the map $\Psi_{x}: \mathbb{R} \longrightarrow M$ defined by

$$
\Psi_{x}(t)=\Psi(t, x), t \in \mathbb{R},
$$

is a $(\delta, T)$-pseudotrajectory of $X . \Psi$ is said to be complete if $\Psi(0, x)=x$ for $x \in M$.

Note that a $(\delta, T)$-method for $X$ can be considered as a family of $(\delta, T)$ pseudotrajectories of $X$.

A method $\Psi$ for $X$ is said to be continuous if the map $\tilde{\Psi}: M \longrightarrow M^{\mathbb{R}}$ given by

$$
\tilde{\Psi}(x)(t)=\Psi(t, x)
$$

for $x \in M$ and $t \in \mathbb{R}$, is continuous under the compact open topology on $M^{\mathbb{R}}$, where $M^{\mathbb{R}}$ denotes the set of all functions from $\mathbb{R}$ into $M$.

The set of all complete $(\delta, 1)$-methods [resp. complete continuous $(\delta, 1)$ methods] for $X \in \mathcal{X}^{1}(M)$ will be denoted by $\mathcal{T}_{a}(\delta, X)$ [resp. $\left.\mathcal{T}_{c}(\delta, X)\right]$. It is clear that if $Y \in \mathcal{X}^{1}(M)$ is another vector field which is sufficiently close to $X$ in the $C^{0}$ topology then it induces a complete continuous method for X.

Let $\mathcal{T}_{h}(\delta, X)$ [resp. $\mathcal{T}_{d}(\delta, X)$ ] be the set of all complete continuous $(\delta, 1)$ methods for $X$ which are induced by $C^{1}$ vector fields $Y$ with $d_{C^{0}}(X, Y)<\delta$ [resp. $d_{C^{1}}(X, Y)<\delta$ ], where $d_{0}$ is the $C^{0}$ metric on $\mathcal{X}^{1}(M)$ such that

$$
d_{C^{0}}(X, Y)=\sup _{x \in M}\{\|X(x)-Y(x)\|\} .
$$

We say that a vector field $X \in \mathcal{X}^{1}(M)$ has the inverse shadowing property with respect to the class $\mathcal{T}_{\alpha}(\alpha=a, c, h, d)$ if for any $\varepsilon>0$ there exists $\delta>0$ such that for any $(\delta, 1)$-method $\Psi \in \mathcal{T}_{\alpha}(\delta, X)$ and any point $x \in M$ there are $y \in M$ and $\alpha_{x} \in \operatorname{Rep}$ for which

$$
d\left(X_{\alpha_{x}(t)}(x), \Psi(t, y)\right)<\varepsilon, t \in \mathbb{R},
$$

where Rep denotes the set of all increasing homeomorphisms $\alpha$ mapping $\mathbb{R}$ onto $\mathbb{R}$ with $\alpha(0)=0($ see $[5,10])$. 


\section{Orbital inverse shadowing}

Definition 3.1. We say that a vector field $X \in \mathcal{X}^{1}(M)$ has the orbital inverse shadowing property with respect to the class $\mathcal{T}_{\alpha}(\alpha=a, c, h, d)$ if for any $\varepsilon>0$ there exists $\delta>0$ such that for any $(\delta, 1)$-method $\Psi \in \mathcal{T}_{\alpha}(\delta, X)$ and any point $x \in M$ there is $y \in M$ for which

$$
d_{H}\left(\overline{O\left(x, X_{t}\right)}, \overline{O(y, \Psi)}\right)<\varepsilon,
$$

where, $d_{H}$ is the Hausdorff metric, $O\left(x, X_{t}\right)=\left\{X_{t}(x): t \in \mathbb{R}\right\}$, and $O(y, \Psi)=$ $\{\Psi(t, y): t \in \mathbb{R}\}$.

Remark 3.2. Let us denote $I S_{\alpha}(M)$ by the set of vector fields on $M$ with the inverse shadowing property with respect to the class $\mathcal{T}_{\alpha}$, and $O I S_{\alpha}(M)$ by the set of vector fields on $M$ with the orbital inverse shadowing property with respect to the class $\mathcal{T}_{\alpha}$, where $\alpha=a, c, h, d$. We denote $I S_{\alpha}^{\circ}(M)$ [resp. $\left.O I S_{\alpha}^{\circ}(M)\right]$ by the $C^{1}$ interior of the set $I S_{\alpha}(M)$ [resp. $\left.O I S_{\alpha}(M)\right]$ in $\mathcal{X}^{1}(M)$, where $\alpha=a, c, h, d$.

Clearly we have the following inclusions:

$$
\begin{array}{lllllll}
I S_{a}(M) & \subset & I S_{c}(M) & \subset & I S_{h}(M) & \subset & I S_{d}(M) \\
O I S_{a}(M) & \subset & \bigcap I S_{c}(M) & \subset & \bigcap_{I} S_{h}(M) & \subset & \bigcap_{I} S_{d}(M) .
\end{array}
$$

A vector field $X \in \mathcal{X}^{1}(M)$ is called structurally stable if there is a $C^{1}$ neighborhood $\mathcal{U}(X)$ of $X \in \mathcal{X}^{1}(M)$ such that every $Y \in \mathcal{U}(X)$ is topologically conjugate to $X$.

It is proved by C. Robinson [21] that if $X \in \mathcal{X}^{1}(M)$ satisfies Axiom A and the strong transversality condition, then $X$ is structurally stable. The inverse implication is the famous "stability conjecture" which is proved for diffeomorphisms by R. Mañé [14] completely and is proved for flows by S. Hayashi [6] and L. Wen [25] completely.

The purpose of this paper is to give a characterization of the structurally stable vector fields via the notion of orbital inverse shadowing.

The main result is the following one.

The Main Theorem. The $C^{1}$ interior of the set of vector fields $X \in \mathcal{X}^{1}(M)$ with the orbital inverse shadowing property with respect to the class $\mathcal{I}_{c}$ (or $\left.\mathcal{T}_{h}, \mathcal{T}_{d}\right)$ coincides with the set of structurally stable vector fields.

We say that $X \in \mathcal{X}^{1}(M)$ is topologically stable in $\mathcal{X}^{1}(M)$ if for any $\varepsilon>0$, there is $\delta>0$ such that for any $Y \in \mathcal{X}^{1}(M)$ with $d_{C^{0}}(X, Y)<\delta$, there is a semiconjugacy $(h, \tau)$ from $Y$ to $X$ satisfying $d(h(x), x)<\varepsilon$ for all $x \in M$.

It follows that if $X \in \mathcal{X}^{1}(M)$ satisfies Axiom A and the strong transversality condition, then $X$ is topologically stable in $X^{1}(M)$ (for more details, see [21] and [15]). As we have pointed out, the structural stable vector fields were characterized as the set of all vector fields satisfying Axiom A and the strong transversality condition. Therefore if $X \in \mathcal{X}^{1}(M)$ is structurally stable then 
it is topologically stable in $\mathcal{X}^{1}(M)$. Furthermore we can easily show that if $X \in \mathcal{X}^{1}(M)$ is topologically stable then it has the inverse shadowing property with respect to the class $\mathcal{T}_{h}$, but the converse does not hold in general as we can see in the below.

Consequently we have the following corollary which is obtained by K. Moriyasu et al. in [15].

Corollary. The $C^{1}$ interior of the set of topologically stable $C^{1}$ vector fields $X \in \mathcal{X}^{1}(M)$ is characterized as the set of all vector fields satisfying Axiom $A$ and the strong transversality condition.

Before giving the proof of the main theorem, we are going to construct a vector field $X \in \mathcal{X}^{1}(M)$ which has the inverse shadowing property with respect to the class $\mathcal{T}_{c}\left(\mathcal{T}_{h}\right)$, but it is not topologically stable.

Our construction is done through the suspension. Let $f$ be a diffeomorphism on $M$. On the set $M \times[0,1]$, we consider the equivalence relation " " defined by $(x, 1) \sim(f(x), 0)$ for $x \in M$. Put $\tilde{M}=M \times[0,1] / \sim$. We define a flow $S_{f}$ on $\tilde{M}$ by putting

$$
S_{f}((x, s), t)=\left(f^{[t+s]}(x), t+s-[t+s]\right)
$$

for $(x, s) \in \tilde{M}$ and $t \in \mathbb{R}$, where $[t]$ denotes the greatest integer less than or equal to $t$. The flow $S_{f}$ on $\tilde{M}$ is called the suspension flow of $f$.

Then we know the following fact: the suspension flow $S_{f}$ of $f$ is topologically stable if and only if $f$ is topologically stable, i.e., for any $\varepsilon>0$, there is $\delta>0$ such that for any $g \in \operatorname{Diff}(M)$ with $d_{C^{0}}(f, g)<\delta$, there is a semiconjugacy $h$ from $g$ to $f$ satisfying $d(h(x), x)<\varepsilon$ for all $x \in M$, where $\operatorname{Diff}(M)$ is the set of diffeomorphisms with the $C^{1}$ topology (for more details, see [24]).

Note that the notion of topological stability of $f \in \operatorname{Diff}(M)$ in the above is slightly different from the original definition of topological stability for homeomorphisms. In the original definition, the perturbation should be a homeomorphism instead of a diffeomorphism. However these two notions are pairwise equivalent if the phase space is the unit circle $S^{1}$. More precisely, the following result can be obtained by the same techniques in [26].

Lemma 3.3. Let $f \in \operatorname{Diff}\left(S^{1}\right)$. Then the followings are pairwise equivalent.

(i) $f$ is topologically conjugate to a Morse-Smale diffeomorphism,

(ii) $f$ is topologically stable,

(iii) for any $\varepsilon>0$, there is $\delta>0$ such that for any $g \in \operatorname{Diff}\left(S^{1}\right)$ with $d_{C^{0}}(f, g)<\delta$, there is a semiconjugacy $h$ from $g$ to $f$ satisfying

$$
d(h(x), x)<\varepsilon \text { for all } x \in S^{1} .
$$

Consider the circle $S^{1}$ with coordinate $x \in[-1,1)$; that is,

$$
x \longleftrightarrow e^{\pi x i}
$$


Let $f$ be a $C^{1}$ diffeomorphism on $S^{1}$ defined by

$$
f(x)= \begin{cases}x+\epsilon x^{4} \sin \frac{\pi}{x} & \text { if } x \neq 0 \\ 0 & \text { if } x=0\end{cases}
$$

where $0<\epsilon<\frac{1}{8}$. Then we can easily check that $f$ has the shadowing property by applying the result in [20]. On the other hand, $f$ is not topologically stable by Lemma 3.3. Then the suspension flow $S_{f}$ of $f$ is not topologically stable by the above properties, and it has the inverse shadowing property with respect to the class $\mathcal{T}_{c}$ by the following two lemmas (Lemmas 3.4 and 3.5). This implies that the concept of topological stability for vector fields is surely stronger than that of inverse shadowing with respect to the class $\mathcal{T}_{c}$.

Lemma 3.4 ([1, Theorem 4.3]). Let $f$ be a diffeomorphism on $M$. The suspension flow $S_{f}$ of $f$ has the inverse shadowing property with respect to the class $\mathcal{T}_{c}\left[\right.$ resp. $\left.\mathcal{T}_{h}\right]$ if and only if $f$ has the inverse shadowing property with respect to the class $\mathcal{T}_{c}\left[\right.$ resp. $\left.\mathcal{T}_{h}\right]$ (for the notion of the inverse shadowing for diffeomorphisms, see [3]).

Lemma 3.5 ([11, Main Theorem]). For any $f \in \operatorname{Diff}\left(S^{1}\right)$, the following statements are pairwise equivalent:

(i) $f$ has the shadowing property,

(ii) $f$ has the inverse shadowing property with respect to the class $\mathcal{T}_{c}$,

(iii) $f$ has the inverse shadowing property with respect to the class $\mathcal{T}_{h}$.

Note that Lemma 3.5 does not hold in general if $\operatorname{dim} M \geq 2$ (see [3, 9]).

Let $\mathcal{X}^{*}(M)$ be the set of $X \in \mathcal{X}^{1}(M)$ with the property that there is a $C^{1}$ neighborhood $\mathcal{U}(X) \subset \mathcal{X}^{1}(M)$ of $X$ such that every singularity and every periodic orbit of $Y \in \mathcal{U}(X)$ are hyperbolic. Denote by $\mathcal{X}^{\sharp}(M)$ the set of vector fields $X \in \mathcal{X}^{*}(M)$ satisfying the following property: there is a $C^{1}$ neighborhood $\mathcal{U}(X) \subset \mathcal{X}^{*}(M)$ of $X$ such that for each $Y \in \mathcal{U}(X)$, the stable manifolds and the unstable manifolds of singularities and periodic orbits of $Y_{t}$ are all transversal. Then we can see that $X \in \mathcal{X}^{\sharp}(M)$ if and only if $X$ satisfies Axiom A and the strong transversality condition (for more details, see [4]).

Recently, Y. Han and K. Lee [5] proved that every structurally stable vector field has the inverse shadowing property with respect to the class $\mathcal{T}_{c}$. Therefore we get the following inclusions:

$$
\mathcal{X}^{\sharp}(M)=S S(M) \subset I S_{c}^{\circ}(M) \subset O I S_{c}^{\circ}(M) \subset O I S_{d}^{\circ}(M),
$$

where $S S(M)$ denotes the set of structurally stable vector fields on $M$.

Consequently, to prove the main theorem, it is enough to show the following theorem. For simplicity, we will denote $O I S_{d}^{\circ}(M)$ by $O I S^{\circ}(M)$.

Theorem 3.6. $O I S^{\circ}(M) \subset \mathcal{X}^{\sharp}(M)$.

The proof of the above theorem is completed by the following three propositions. The techniques in our proof are similar to those in [15]. 
Throughout this paper, let $\operatorname{Sing}(X)$ be the set of all singularities of $X$, and let $P O\left(X_{t}\right)$ be the set of all periodic orbits (which are not singularities) of the generated flow $X_{t}$.

Proposition A. $O I S^{\circ}(M) \subset \mathcal{X}^{*}(M)$.

Proposition B. Let $X \in O I S^{\circ}(M), p \in \operatorname{Sing}(X)$ and $q \in \operatorname{Sing}(X) \cup P O\left(X_{t}\right)$. Then the stable manifold of $p$ and the unstable manifold of $q$ are transverse.

Proposition C. Let $X \in O I S^{\circ}(M)$ and let $\gamma, \gamma^{\prime} \in P O\left(X_{t}\right)$. Then the stable manifold of $\gamma$ and the unstable manifold of $\gamma^{\prime}$ are transverse.

\section{Auxiliary lemmas}

We say that $p \in \operatorname{Sing}(X)$ is hyperbolic if the linear map $D_{p} X: T_{p} M \longrightarrow T_{p} M$ has no eigenvalue $\lambda$ with $\operatorname{Re}(\lambda)=0$. For a hyperbolic singularity $p$, we define the stable manifold $W^{s}\left(p, X_{t}\right)$ and the unstable manifold $W^{u}\left(p, X_{t}\right)$ of $p$ as following;

$$
\begin{gathered}
W^{s}\left(p, X_{t}\right)=\left\{x \in M: d\left(X_{t}(x), p\right) \rightarrow 0 \text { as } t \rightarrow \infty\right\}, \\
W^{u}\left(p, X_{t}\right)=\left\{x \in M: d\left(X_{t}(x), p\right) \rightarrow 0 \text { as } t \rightarrow-\infty\right\} .
\end{gathered}
$$

A point $x \in M$ is called a non-wandering point of $X$ if for any neighborhood $U$ of $x$ in $M$, there is $t \geq 1$ such that $X_{t}(U) \cap U \neq \emptyset$. The set of all non-wandering points of $X$ is denoted by $\Omega\left(X_{t}\right)$. Clearly, $\operatorname{Sing}(X) \cup P O\left(X_{t}\right) \subset \Omega\left(X_{t}\right)$.

Hereafter, we assume that the exponential map $\exp _{p}: T_{p} M(1) \longrightarrow M$ is well defined for all $p \in M$, where $T_{p} M(1)=\left\{v \in T_{p} M:\|v\| \leq 1\right\}$. Let $B_{\varepsilon}(x)=$ $\{y \in M: d(x, y) \leq \varepsilon\}(\varepsilon>0)$. To prove the main theorem, we need the following three lemmas in [15].

Lemma 4.1 ([15, Lemma 1.1]). Let $X \in \mathcal{X}^{1}(M)$ and $p \in \operatorname{Sing}(X)$. Then for every $C^{1}$ neighborhood $\mathcal{U}(X) \subset \mathcal{X}^{1}(M)$ of $X$, there are $\delta_{0}>0$ and $\varepsilon_{0}>0$ such that if $O_{\delta}: T_{p} M \longrightarrow T_{p} M$ is a linear map with $\left\|O_{\delta}-D_{p} X\right\|<\delta<\delta_{0}$, then there is $Y^{\delta} \in \mathcal{U}(X)$ satisfying

$$
Y^{\delta}(x)= \begin{cases}\left(D_{\exp _{p}^{-1}(x)} \exp _{p}\right) \circ O_{\delta} \circ \exp _{p}^{-1}(x) & \text { if } x \in B_{\varepsilon_{0} / 4}(p), \\ X(x) & \text { if } x \notin B_{\varepsilon_{0}}(p) .\end{cases}
$$

Furthermore, $d_{C^{1}}\left(Y^{\delta}, Y^{0}\right) \longrightarrow 0$ as $\delta \longrightarrow 0$. Here $Y^{0}$ is vector field for $O_{\delta}=$ $D_{p} X$.

Let $X \in \mathcal{X}^{1}(M)$. For every $x \in M \backslash \operatorname{Sing}(X)$, put

$$
\hat{\Pi}_{x}=(\operatorname{Span} X(x))^{\perp} \subset T_{x} M, \Pi_{x, r}=\exp _{x}\left(\hat{\Pi}_{x, r}\right) \text { and } \Pi_{x}=\Pi_{x, 1},
$$

where, $\hat{\Pi}_{x, r}=\left\{v \in \hat{\Pi}_{x}:\|v\|<r\right\}$ for $r>0$. Then, for given $x^{\prime}=X_{t_{0}}(x)\left(t_{0}>\right.$ $0)$, there are $r_{0}>0$ and a $C^{1} \operatorname{map} \tau: \Pi_{x, r_{0}} \longrightarrow \mathbb{R}$ such that

$$
X_{\tau(y)}(y) \in \Pi_{x^{\prime}}\left(y \in \Pi_{x, r_{0}}\right) \text { and } \tau(x)=t_{0} .
$$


The flow $X_{t}$ uniquely defines the Poincaré map $f: \Pi_{x, r_{0}} \longrightarrow \Pi_{x^{\prime}}$ by

$$
f(y)=X_{\tau(y)}(y) \text { for } y \in \Pi_{x, r_{0}} .
$$

The map is $C^{1}$ embedding whose image is interior to $\Pi_{x^{\prime}}$ if $r_{0}$ is small. We denote the set of all $C^{1}$ embeddings from $\Pi_{x, r}$ to $\Pi_{x^{\prime}}(r>0)$ by $\operatorname{Emb}^{1}\left(\Pi_{x, r}, \Pi_{x^{\prime}}\right)$ and topologize it by using the $C^{1}$ topology. If $X_{t}(x) \neq x$ for $0<t \leq t_{0}$ and $r_{0}$ is sufficiently small, then $(t, y) \mapsto X_{t}(y) C^{1}$ embeds

$$
\left\{(t, y) \in \mathbb{R} \times \Pi_{x, r}: 0 \leq t \leq \tau(y)\right\}
$$

for $0<r \leq r_{0}$. The image

$$
\left\{X_{t}(y): y \in \Pi_{x, r} \text { and } 0 \leq \mathrm{t} \leq \tau(\mathrm{y})\right\}
$$

is called a $t_{0}$-time length flow box and is denoted by $F_{x}\left(X_{t}, r, t_{0}\right)$. For $\varepsilon>0$, let $\mathcal{N}_{\varepsilon}\left(\Pi_{x, r}\right)$ be the set of all diffeomorphisms $\varphi: \Pi_{x, r} \longrightarrow \Pi_{x, r}$ such that

$$
\operatorname{supp}(\varphi) \subset \Pi_{x, \frac{r}{2}} \text { and } d_{C^{1}}(\varphi, i d)<\varepsilon .
$$

Here $d_{C^{1}}$ is the usual $C^{1}$ metric, $i d: \Pi_{x, r} \longrightarrow \Pi_{x, r}$ is the identity map and the support of $\varphi$ is the closure of the set where it differs from $i d$.

Lemma 4.2 ([15, Lemma 1.2]). Let $X \in \mathcal{X}^{1}(M)$. Suppose $X_{t}(x) \neq x$ for $0<t \leq t_{0}(x \notin \operatorname{Sing}(X))$, and let $f: \Pi_{x, r_{0}} \longrightarrow \Pi_{x^{\prime}}\left(x^{\prime}=X_{t_{0}}(x)\right)$ be the Poincaré map ( $r_{0}>0$ is sufficiently small). Then, for every $C^{1}$ neighborhood $\mathcal{U}(X) \subset \mathcal{X}^{1}(M)$ of $X$ and $0<r \leq r_{0}$, there is $\varepsilon>0$ with the property that for every $\varphi \in \mathcal{N}_{\varepsilon}\left(\Pi_{x, r}\right)$, there exists $Y \in \mathcal{U}(X)$ satisfying

$$
\begin{cases}Y(y)=X(y) & \text { if } y \notin F_{x}\left(X_{t}, r, t_{0}\right) \\ f_{Y}(y)=f \circ \varphi(y) & \text { if } y \in \Pi_{x, r} .\end{cases}
$$

Here $f_{Y}: \Pi_{x, r} \longrightarrow \Pi_{x^{\prime}}$ is the Poincaré map defined by $Y_{t}$.

Remark 4.3. Under the same notation and assumption of Lemma 4.2, let $Y^{\delta} \in$ $\mathcal{U}(X)$ be given by Lemma 4.2 for $\varphi_{\delta} \in N_{\varepsilon\left(\Pi_{x, r}\right)}(\delta>0)$. If $\varphi_{\delta} \longrightarrow \varphi$ as $\delta \longrightarrow 0$ with respect to the $C^{1}$ topology, then by the construction of $Y^{\delta}$, we have $d_{C^{1}}\left(Y^{\delta}, Y\right) \longrightarrow 0$ as $\delta \longrightarrow 0$.

Let $X \in \mathcal{X}^{1}(M)$ and suppose $p \in \gamma \in P O\left(X_{t}\right)\left(X_{T}(p)=p, T>0\right)$. If $f: \Pi_{p, r_{0}} \longrightarrow \Pi_{p}$ is the Poincaré map $\left(r_{0}>0\right)$, then $f(p)=p$. We say that $\gamma$ is hyperbolic if $p$ is a hyperbolic fixed point of $f$. If $\gamma \in P O\left(X_{t}\right)$ is hyperbolic, then the stable manifold $W^{s}\left(\gamma, X_{t}\right)$ and the unstable manifold $W^{u}\left(\gamma, X_{t}\right)$ of $\gamma$ are defined by the usual way. Let $\gamma, \gamma^{\prime} \in P O\left(X_{t}\right)$ be hyperbolic. We say that $\gamma$ is transverse to $\gamma^{\prime}$ if for any $x \in W^{s}\left(\gamma, X_{t}\right) \cap W^{u}\left(\gamma^{\prime}, X_{t}\right)$,

$$
T_{x} M=T_{x} W^{s}\left(\gamma, X_{t}\right)+T_{x} W^{u}\left(\gamma^{\prime}, X_{t}\right) \text {. }
$$

Lemma 4.4 ([15, Lemma 1.3]). Let $X \in \mathcal{X}^{1}(M), p \in \gamma \in P O\left(X_{t}\right)\left(X_{T}(p)=\right.$ p) and $f: \Pi_{p, r_{0}} \longrightarrow \Pi_{p}$ be as above, and let $\mathcal{U}(X) \subset \mathcal{X}^{1}(M)$ be a $C^{1}$ neighborhood of $X$ and $0<r \leq r_{0}$ be given. Then there are $\delta_{0}>0$ and $0<\varepsilon_{0}<\frac{r}{2}$ such that for a linear isomorphism $O_{\delta}: \hat{\Pi}_{p} \longrightarrow \hat{\Pi}_{p}$ with $\left\|O_{\delta}-D_{p} f\right\|<\delta<\delta_{0}$, there is $Y^{\delta} \in \mathcal{U}(X)$ satisfying 
(i) $Y^{\delta}(x)=X(x)$ if $x \notin F_{p}\left(X_{t}, r, T\right)$,

(ii) $p \in \gamma \in P O\left(Y_{t}^{\delta}\right)$,

(iii)

$$
g_{Y^{\delta}}(x)=\left\{\begin{array}{lll}
\exp _{p} \circ O_{\delta} \circ \exp _{p}^{-1}(x) & \text { if } x \in B_{\varepsilon_{0} / 4}(p) \cap \Pi_{p, r} \\
f(x) & \text { if } x \notin B_{\varepsilon_{0}}(p) \cap \Pi_{p, r},
\end{array}\right.
$$

where $g_{Y^{\delta}}: \Pi_{p, r} \longrightarrow \Pi_{p}$ is the Poincaré map of $Y_{t}^{\delta}$. Furthermore, let $Y^{0}$ be the vector field for $O_{\delta}=D_{p} f$. Then we have

(iv) $d_{C^{1}}\left(Y^{\delta}, Y^{0}\right) \longrightarrow 0$ as $\delta \longrightarrow 0$.

\section{Proof of the main theorem}

In this section, we will prove Propositions A-C.

Proof of Proposition A. The proof is similar to that of Proposition A in [15], but for the sake of completeness we present it here. The proof is divided into two cases.

Case 1. We prove the proposition for singularities.

Let $X \in O I S^{\circ}(M)$. Suppose that there is an eigenvalue $\lambda$ of $D_{p} X$ with $\operatorname{Re}(\lambda)=0$ for some $p \in \operatorname{Sing}(X)$. By Lemma 4.1, for any $C^{1}$ neighborhood $\mathcal{U}(X) \subset O I S^{\circ}(M)$ of $X$, there are $\delta_{0}>0$ and $\varepsilon_{0}>0$ such that for every linear isomorphism $O_{\delta}: T_{p} M \longrightarrow T_{p} M$ with $\left\|O_{\delta}-D_{p} X\right\|<\delta<\delta_{0}$, there is $Y^{\delta} \in \mathcal{U}(X)$ satisfying

$$
Y^{\delta}(x)=\left\{\begin{array}{lll}
\left(D_{\exp _{p}^{-1}(x)} \exp _{p}\right) \circ O_{\delta} \circ \exp _{p}^{-1}(x) & \text { if } x \in B_{\varepsilon_{0} / 4}(p) \\
X(x) & \text { if } x \notin B_{\varepsilon_{0}}(p) .
\end{array}\right.
$$

Let $Y^{0} \in \mathcal{U}(X)$ be as above for $O_{0}=D_{p} X$ and denote $Y^{0}$ by $Y$. For $0<\varepsilon<\frac{\varepsilon_{0}}{16}$, let $0<\delta<\min \left\{\delta_{0}, \varepsilon\right\}$ be as in the definition of $O I S(M)$ of $Y_{t}$. Pick $0<\delta^{\prime}<\delta$ and a linear isomorphism $O_{\delta^{\prime}}: T_{p} M \longrightarrow T_{p} M$ whose any eigenvalue has a non-zero real part such that if $\left\|O_{\delta^{\prime}}-D_{p} X\right\|<\delta^{\prime}$, then $d_{C^{1}}\left(Y^{\delta^{\prime}}, Y\right)<\delta$. Then $Y_{t}^{\delta^{\prime}} \in \mathcal{T}_{d}(\delta, Y)$ and $p$ is a hyperbolic singularity of $Y^{\delta^{\prime}}$. The restriction $\left.Y_{t}^{\delta^{\prime}}\right|_{B_{\varepsilon_{0} / 4}(p)}$ can be regarded as the flow induced from the hyperbolic linear vector field $\left.O_{\delta^{\prime}}\right|_{\exp _{p}^{-1}\left(B_{\varepsilon_{0} / 4}(p)\right)}$ with respect to the exponential coordinates. Since $d_{C^{1}}\left(Y, Y^{\delta^{\prime}}\right)<\delta$, for any $x \in M$, there is a point $y \in M$ such that

$$
d_{H}\left(\overline{O\left(x, Y_{t}\right)}, \overline{O\left(y, Y_{t}^{\delta^{\prime}}\right)}\right)<\varepsilon .
$$

By the existence of the $\lambda$, we can take $z \in M$ such that $p \notin B_{\varepsilon}\left(O\left(z, Y_{t}\right)\right)$ $\subset B_{\varepsilon_{0} / 8}(p)$ (by reducing $\varepsilon$ if necessary). Here $B_{\varepsilon}(A)=\bigcup_{x \in A} B_{\varepsilon}(x)$ for $A \subset$ $M$. Let $w$ be an orbital inverse shadowing point of $O\left(z, Y_{t}\right)$. Then we have $O\left(w, Y_{t}^{\delta^{\prime}}\right) \subset B_{\varepsilon}\left(O\left(z, Y_{t}\right)\right)$. Since $\left.Y_{t}^{\delta^{\prime}}\right|_{B_{\varepsilon_{0} / 4}(p)}$ is regarded as the flow induced from the hyperbolic linear vector field $\left.O_{\delta^{\prime}}\right|_{\exp _{p}^{-1}\left(B_{\varepsilon_{0} / 4}(p)\right)}$, there is $t \in \mathbb{R}$ such that $Y_{t}^{\delta^{\prime}}(w) \notin B_{\varepsilon_{0} / 8}(p)$, which contradicts to $B_{\varepsilon}\left(O\left(z, Y_{t}\right)\right) \subset B_{\varepsilon_{0} / 8}(p)$. 
Case 2. We prove the proposition for periodic orbits.

Let $\mathcal{U}(X) \subset O I S^{\circ}(M)$ be a $C^{1}$ neighborhood of $X$ and pick $p \in \gamma \in$ $P O\left(X_{t}\right)\left(X_{T}(p)=p, T>0\right)$. The flow $X_{t}$ defines the Poincaré map $f$ : $\Pi_{p, r_{0}} \longrightarrow \Pi_{p}$, (for some $r_{0}>0$ ). By assuming that there is an eigenvalue $\lambda$ of $D_{p} f$ with $|\lambda|=1$, we shall derive a contradiction. Let $\delta_{0}>0$ and $0<\varepsilon_{0}<r_{0}$ be given by Lemma 4.4 for the $\mathcal{U}(X)$. Then, for every linear isomorphism $O_{\delta}: \hat{\Pi}_{p} \longrightarrow \hat{\Pi}_{p}$ with $\left\|O_{\delta}-D_{p} f\right\|<\delta<\delta_{0}$, there is $Y^{\delta} \in \mathcal{U}(X)$ such that

- $Y^{\delta}(x)=X(x) \quad$ if $\quad x \notin F_{p}\left(X_{t}, r_{0}, T\right)$,

- $g_{Y^{\delta}}(x)= \begin{cases}\exp _{p} \circ O_{\delta} \circ \exp _{p}^{-1}(x) & \text { if } x \in B_{\varepsilon_{0} / 4}(p) \cap \Pi_{p, r_{0}} \\ f(x) & \text { if } x \notin B_{\varepsilon_{0}}(p) \cap \Pi_{p, r_{0}},\end{cases}$

- $d_{C^{1}}\left(Y^{\delta}, Y^{0}\right) \longrightarrow 0$ as $\delta \longrightarrow 0$.

Denote $Y^{0}$ by $Y$. For $0<\varepsilon<\frac{\varepsilon_{0}}{16}$, let $0<\delta<\min \left\{\delta_{0}, \varepsilon\right\}$ be as in the definition of $O I S(M)$ of $Y_{t}$. Take a $0<\delta^{\prime}<\delta$ and a hyperbolic linear isomorphism $O_{\delta^{\prime}}: \hat{\Pi}_{p} \longrightarrow \hat{\Pi}_{p}$ such that if $\left\|O_{\delta^{\prime}}-D_{p} X\right\|<\delta^{\prime}$, then $d_{C^{1}}\left(Y, Y^{\delta}\right)<\delta$. Then $Y_{t}^{\delta^{\prime}} \in \mathcal{T}_{d}(\delta, Y)$ and $\gamma$ is a hyperbolic periodic orbit of $Y_{t}^{\delta^{\prime}}$. Therefore for any $x \in M$, there is $y \in M$ such that

$$
d_{H}\left(\overline{O\left(x, Y_{t}\right)}, \overline{O\left(y, Y_{t}^{\delta^{\prime}}\right)}\right)<\varepsilon .
$$

Note that $g_{Y^{\delta^{\prime}}}(p)=p$ and the restriction $\left.g_{Y^{\delta^{\prime}}}\right|_{B_{\varepsilon_{0} / 4}(p) \cap \Pi_{p, r_{0}}}$ is regarded as

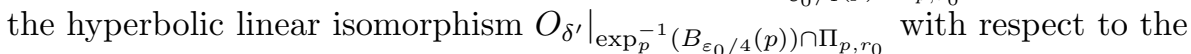
exponential coordinates. Since $|\lambda|=1$, we may take $z \in \Pi_{p, r_{0}}$ such that

$$
p \notin B_{\varepsilon}\left(\left\{g_{Y}^{i}(z): i \in \mathbb{Z}\right\}\right) \subset B_{\varepsilon_{0} / 8}(p) \cap \Pi_{p, r_{0}} .
$$

Let $w$ be an orbital inverse shadowing point of $O\left(z, Y_{t}\right)$. Set $w^{\prime}=Y_{t^{\prime}}^{\delta^{\prime}}(w) \in$ $\Pi_{p, r_{0}}$, where $\left|t^{\prime}\right|=\min \left\{|t|: Y_{t}^{\delta^{\prime}}(w) \in \Pi_{p, r_{0}}\right\}$. Since

$$
d_{H}\left(\overline{O\left(z, Y_{t}\right)}, \overline{O\left(w, Y_{t}^{\delta^{\prime}}\right)}\right)<\varepsilon
$$

we have $d\left(g_{Y^{\delta^{\prime}}}^{i}\left(w^{\prime}\right), Y_{t}(z)\right)<\varepsilon$ for some $t \in \mathbb{R}$. By the fact that $g_{Y^{\delta^{\prime}}}$ is the Poincaré map of the vector field $Y^{\delta^{\prime}}$ with $d_{C^{1}}\left(Y, Y^{\delta^{\prime}}\right)<d$, we can choose a sequence $\left\{g_{Y^{\delta^{\prime}}}^{i}\left(w^{\prime}\right)\right\} \subset \Pi_{p, r_{0}}$ such that $d\left(g_{Y^{\delta^{\prime}}}^{i}\left(w^{\prime}\right), g_{Y}^{i}(z)\right)<\varepsilon$ for all $i \in$ $\mathbb{Z}$. Since $\left.g_{Y^{\delta^{\prime}}}\right|_{B_{\varepsilon_{0} / 4}(p) \cap \Pi_{p, r_{0}}}$ is regarded as the hyperbolic linear isomorphism $\left.O_{\delta^{\prime}}\right|_{\exp _{p}^{-1}\left(B_{\varepsilon_{0} / 4}(p)\right) \cap \Pi_{p, r_{0}}}$, there is $k$ such that $g_{Y^{\delta^{\prime}}}^{k}\left(w^{\prime}\right) \notin B_{\varepsilon_{0} / 8}(p) \cap \Pi_{p, r_{0}}$, which contradicts to $B_{\varepsilon}\left(\left\{g_{Y}^{i}(z): i \in \mathbb{Z}\right\}\right) \subset B_{\varepsilon_{0} / 8}(p) \cap \Pi_{p, r_{0}}$.

Before the proof of Proposition B, we give a lemma.

Lemma 5.1. Let $X \in \mathcal{X}^{1}(M)$. Suppose that $p \in \operatorname{Sing}(X) \cup P O\left(X_{t}\right)$ and $q \in$ $\operatorname{Sing}(X) \cup P O\left(X_{t}\right)$ are hyperbolic, and $p \neq q$. Let $x \in W^{s}\left(p, X_{t}\right) \cap W^{u}\left(q, X_{t}\right)$. Then there exist $\varepsilon^{\star}>0$ and $\eta^{\star}>0$ such that for any $y \in B_{\eta^{\star}}(x)-\Delta_{s}[$ resp. $\left.y \in B_{\eta^{\star}}(x)-\Delta_{u}\right]$, there exists $t^{\prime}>0\left[\right.$ resp. $\left.t^{\prime}<0\right]$ satisfying

$$
\left.\inf \left\{d\left(X_{t^{\prime}}(y), z\right): z \in \overline{O\left(x, X_{t}\right.}\right)\right\} \geq \varepsilon^{\star} .
$$


Here $\Delta_{s}\left[\right.$ resp. $\left.\Delta_{u}\right]$ is the connected component of $W^{s}\left(p, X_{t}\right)\left[\right.$ resp. $\left.W^{u}\left(q, X_{t}\right)\right]$ in $B_{\eta^{\star}}(x)$ containing $x$.

Proof. We will only prove the case that $q$ and $p$ are singularities. The proof of the other cases are similar.

Let $r_{0}>0$ be a small constant such that $B_{r_{0}}(p)$ is a local chart about $p$. Let $C^{u}\left(r_{0} / 2\right)=\left\{B_{r_{0}}(p)-B_{r_{0} / 2}(p)\right\} \cap W_{l o c}^{u}(p)$. Since $x \in W^{s}\left(p, X_{t}\right) \cap W^{u}\left(q, X_{t}\right)$, we have

$$
C^{u}\left(r_{0} / 2\right) \cap \overline{O\left(x, X_{t}\right)}=\emptyset .
$$

Thus

$$
d_{H}\left(C^{u}\left(r_{0} / 2\right), \overline{O\left(x, X_{t}\right)}\right)>0 .
$$

Set $\varepsilon_{1}=d_{H}\left(C^{u}\left(r_{0} / 2\right), \overline{O\left(x, X_{t}\right)}\right) / 2$. By the hyperbolicity of $p$, there exists a neighborhood $B_{\eta_{1}}(x)$ of $x$ such that for any $y \in B_{\eta_{1}}(x)-\Delta_{s}$, there exists $t^{\prime}>0$ with $X_{t^{\prime}}(y) \in N_{\varepsilon_{1}}\left(C^{u}\left(r_{0} / 2\right)\right)$. Thus we have

$$
\begin{aligned}
d_{H}\left(X_{t^{\prime}}(y), \overline{O\left(x, X_{t}\right)}\right) & \geq d_{H}\left(C^{u}\left(r_{0} / 2\right), \overline{O\left(x, X_{t}\right)}\right)-d_{H}\left(X_{t^{\prime}}(y), C^{u}\left(r_{0} / 2\right)\right) \\
& \geq 2 \varepsilon_{1}-\varepsilon_{1}=\varepsilon_{1} .
\end{aligned}
$$

Similarly we can choose $\varepsilon_{2}>0$ and $\eta_{2}>0$ such that for any $y \in B_{\eta_{2}}(x)-\Delta_{u}$, there exists $t^{\prime}<0$ satisfying

$$
d_{H}\left(X_{t^{\prime}}(y), \overline{O\left(x, X_{t}\right.}\right) \geq \varepsilon_{2} .
$$

Let $\varepsilon^{\star}=\min \left\{\varepsilon_{1}, \varepsilon_{2}\right\}$ and $\eta^{\star}=\min \left\{\eta_{1}, \eta_{2}\right\}$. It is easy to see that $\varepsilon^{\star}$ and $\eta^{\star}$ satisfy the conclusion.

Let us start the proof of Proposition B.

Proof of Proposition B. Let $X \in O I S^{\circ}(M)$. Suppose that $x \in W^{s}\left(p, X_{t}\right) \cap$ $W^{u}\left(q, X_{t}\right), p \in \operatorname{Sing}(X), q \in \operatorname{Sing}(X) \cup P O\left(X_{t}\right)$ and $T_{x} M \neq T_{x} W^{s}\left(p, X_{t}\right)+$ $T_{x} W^{u}\left(q, X_{t}\right)$. We may assume that $x$ is very near $p$. Take $r_{0}>0$ small enough so that

- there are the Poincaré maps $f: \Pi_{x, r_{0}} \longrightarrow \Pi_{X_{1}(x)}$ and $f^{\prime}: \Pi_{X_{-1}(x), r_{0}} \longrightarrow \Pi_{x}$,

- $\left\{X_{t}(x): t<0\right\} \cap \Pi_{x, r_{0}}=\emptyset$ and $\left\{X_{t}: t<-1\right\} \cap \Pi_{X_{-1}(x), r_{0}}=\emptyset$,

- $W_{2 r_{0}}^{u}\left(q, X_{t}\right) \cap\left(\Pi_{x, r_{0}} \cup \Pi_{X_{-1}(x), r_{0}}\right)=\emptyset$,

where $W_{2 r_{0}}^{u}\left(q, X_{t}\right)$ is the local unstable manifold of $q$. Let $V^{s}(x)$ be the connected component of $W^{s}\left(p, X_{t}\right) \cap \Pi_{x}$ containing $x$, and $V^{u}(x)$ be the connected component of $W^{u}\left(q, X_{t}\right) \cap \Pi_{x}$ containing $x$.

Clearly, we have

$$
0 \leq \operatorname{dim} V^{s}(x) \leq \operatorname{dim} \Pi_{x} \text { and } 0 \leq \operatorname{dim} V^{u}(x) \leq \operatorname{dim} \Pi_{x} .
$$

If $\operatorname{dim} V^{s}(x)=\operatorname{dim} \Pi_{x}$ or $\operatorname{dim} V^{u}(x)=\operatorname{dim} \Pi_{x}$, then there is nothing to prove.

Notice that $\hat{\Pi}_{x} \neq T_{x} V^{s}(x)+T_{x} V^{u}(x)$. We shall divide the proof into the following two cases:

Case (1.1) $\operatorname{dim} V^{s}(x)+\operatorname{dim} V^{u}(x)<\operatorname{dim} \Pi_{x}$, 
Case (1.2) $\operatorname{dim} V^{s}(x)+\operatorname{dim} V^{u}(x) \geq \operatorname{dim} \Pi_{x}$.

Proof for Case (1.1). Let $V_{r}^{u}(x)$ be the connected component of $V^{u}(x) \cap$ $B_{r}(x)$ containing $x$, and $V_{r}^{s}(x)$ be the connected component of $V^{s}(x) \cap B_{r}(x)$ containing $x$ for $r>0$. Choose $0<r^{\prime}<\frac{r_{0}}{4}$ with the following properties:

- for every $\delta>0$, there exists $\varphi_{\delta} \in N_{\varepsilon(\delta)}\left(\Pi_{x, r_{0}}\right)$ satisfying

$$
\varphi_{\delta}\left(V_{r^{\prime}}^{u}(x)\right) \cap V^{s}(x)=\emptyset,
$$

- $V_{r^{\prime}}^{u}(x)=\left[\bigcup_{0 \leq t \leq t_{1}} X_{t}\left(W_{2 r_{0}}^{u}\left(q, X_{t}\right)\right)\right] \cap \Pi_{x, r_{0}} \cap B_{r^{\prime}}(x)$, where $t_{1}>0$ is a constant with $X_{-t_{1}}(x) \in W_{r_{0}}^{u}\left(q, X_{t}\right)$,

- $V_{r^{\prime}}^{s}(x)=\left[\bigcup_{0 \leq t \leq t_{2}} X_{-t}\left(W_{2 r_{0}}^{s}\left(p, X_{t}\right)\right)\right] \cap \Pi_{x, r_{0}} \cap B_{r^{\prime}}(x)$, where $t_{2}>0$ is a constant with $X_{t_{2}}(x) \in W_{r_{0}}^{s}\left(p, X_{t}\right)$,

- $B_{r^{\prime}}\left(\left\{X_{t}(x): t \leq 0\right\}\right) \cap \Pi_{x, r_{0}}=B_{r^{\prime}}(x) \cap \Pi_{x, r_{0}}$.

For any small enough $\delta>0$, we can choose a vector field $Y^{\delta}$ given by Lemma 4.2 for the perturbation (2); i.e., $Y^{\delta}$ satisfies the followings:

$$
\begin{cases}Y^{\delta}(y)=X(y) & \text { if } y \notin F_{x}\left(X_{t}, r_{0}, 1\right), \\ g(y)=f \circ \varphi_{\delta}(y) & \text { if } y \in \Pi_{x, r_{0}}, \\ d_{C^{1}}\left(X, Y^{\delta}\right)<\delta, & \end{cases}
$$

where $g: \Pi_{x, r_{0}} \longrightarrow \Pi_{X_{1}(x)}$ is the Poincaré map induced by $Y_{t}^{\delta}$. We may assume that $F_{x}\left(Y_{t}^{\delta}, r_{0}, 1\right) \cap W_{r_{0}}^{s}\left(p, Y_{t}^{\delta}\right)=\emptyset$ for sufficiently small $r_{0}$.

For the vector field $X$ and a point $x \in W^{s}\left(p, X_{t}\right) \cap W^{u}\left(q, X_{t}\right)$, we choose two numbers $\varepsilon^{\star}>0$ and $\eta^{\star}>0$ satisfying the conclusion of Lemma 5.1. Take $\varepsilon>0$ with $\varepsilon<\min \left\{\frac{\varepsilon^{\star}}{2}, \frac{\eta^{\star}}{2}, \frac{r^{\prime}}{2}\right\}$. Let $0<\delta=\delta(\varepsilon)<\varepsilon$ be the corresponding number with respect to $\varepsilon$ in the definition of the orbital inverse shadowing of $X$. Then the vector field $Y^{\delta}$ constructed as the above is an element of $\mathcal{T}_{d}\left(\delta, X_{t}\right)$. For simplicity, we denote $Y^{\delta}$ by $Y$. Then there is $w \in M$ such that

$$
d_{H}\left(\overline{O\left(x, X_{t}\right)}, \overline{O\left(w, Y_{t}\right)}\right)<\varepsilon .
$$

Since $X(y)=Y(y)$ for $y \notin F_{x}\left(X_{t}, r_{0}, 1\right), p$ is a singularity and $q$ is also a singularity or a periodic orbit of $Y_{t}$. Thus the $Y_{t^{-}}$orbit of $w$ passes through $B_{\eta^{\star}}(x)$. Take a point $w^{\prime} \in O\left(w, Y_{t}\right) \cap B_{\eta^{\star}}(x) \cap \Pi_{x, r_{0}}$. If $w^{\prime} \in V_{r^{\prime}}^{u}(x)$, then by the construction of $Y$ and by Lemma 5.1, we can find $t^{\prime}>0$ such that

$$
\inf \left\{d\left(Y_{t}\left(w^{\prime}\right), z\right): z \in \overline{O\left(x, X_{t}\right)}\right\} \geq \varepsilon^{\star} .
$$

Therefore we have

$$
d_{H}\left(\overline{O\left(w, Y_{t}\right)}, \overline{O\left(x, X_{t}\right)}\right) \geq \varepsilon^{\star}>\varepsilon .
$$

If $w^{\prime}$ is not in $V_{r_{0}}^{u}(x)$, then similarly we have

$$
d_{H}\left(\overline{O\left(x, X_{t}\right)}, \overline{O\left(w, Y_{t}\right)}\right) \geq \varepsilon^{\star}>\varepsilon .
$$

Consequently we get a contradiction, and so complete of the proof of Case (1.1).

Proof for Case (1.2). Take a $C^{1}$ neighborhood $\mathcal{U}(X) \subset O I S(M)$ of $X$. Let $V^{s}\left(X_{-1}(x)\right)$ [resp. $\left.V^{u}\left(X_{-1}(x)\right)\right]$ be the connected component of $W^{s}\left(p, X_{t}\right) \cap$ 
$\Pi_{X_{-1}(x)}\left[\right.$ resp. $\left.W^{u}\left(q, X_{t}\right) \cap \Pi_{X_{-1}(x)}\right]$ containing $X_{-1}(x)$. Let $f^{\prime}: \Pi_{X_{-1}(x), r_{0}}$ $\longrightarrow \Pi_{x}$ be the Poincaré map. For the above $\mathcal{U}(X)$, let $\mu=\mu(\mathcal{U}(X))>0$ be given by Lemma 4.2. Since

$$
\hat{\Pi}_{x} \neq T_{x} V^{s}(x)+T_{x} V^{u}(x) \text { and } \operatorname{dim} V^{s}(x)+\operatorname{dim} V^{u}(x) \geq \operatorname{dim} \Pi_{x},
$$

there are $0<r_{1}<\frac{r_{0}}{4}, \tilde{\varphi} \in \mathcal{N}_{\mu}\left(\Pi_{X_{-1}(x), r_{0}}\right)$ and a submanifold $V\left(X_{-1}(x)\right) \subset$ $\Pi_{X-1}(x), r_{0}$ such that

- $V^{s}\left(X_{-1}(x)\right) \cap B_{r_{1}}\left(X_{-1}(x)\right) \subset V\left(X_{-1}(x)\right)$

- $\tilde{\varphi}\left(V^{u}\left(X_{-1}(x)\right) \cap B_{r_{1}}\left(X_{-1}(x)\right)\right) \subset V\left(X_{-1}(x)\right)$ and $\tilde{\varphi}\left(X_{-1}(x)\right)=X_{-1}(x)$,

- $\operatorname{dim} V^{s}(x)+\operatorname{dim} V^{u}(x)-\operatorname{dim} \Pi_{x}<\operatorname{dim} V\left(X_{-1}(x)\right)<\operatorname{dim} \Pi_{X_{-1}(x)}$.

Let $Y \in \mathcal{U}(X)$ and $g=f^{\prime} \circ \tilde{\varphi}: \Pi_{X_{-1}(x), r_{0}} \longrightarrow \Pi_{x}$ (since $g\left(X_{-1}(x)\right)=x$ ) be given by Lemma 4.2. Let $V^{s}\left(x, Y_{t}\right)$ [resp. $V^{u}\left(x, Y_{t}\right)$ ] be the connected component of $W^{s}\left(p, Y_{t}\right) \cap \Pi_{x}$ [resp. $W^{u}\left(q, Y_{t}\right) \cap \Pi_{x}$ ] containing $x$. If we put $V\left(x, Y_{t}\right)=f^{\prime}\left(V\left(X_{-1}(x)\right)\right)$, then we get $\operatorname{dim} V\left(x, Y_{t}\right)<\operatorname{dim} \Pi_{x}$. It is easy to choose $0<r_{2}<\frac{r_{0}}{4}$ satisfying

$$
V^{u}\left(x, Y_{t}\right) \cap B_{r_{2}}(x) \subset V\left(x, Y_{t}\right) \text { and } V^{s}\left(x, Y_{t}\right) \cap B_{r_{2}}(x) \subset V\left(x, Y_{t}\right) .
$$

By the choice of $r_{0}$, we can see that

- the map $f: \Pi_{x, r_{0}} \longrightarrow \Pi_{X_{1}(x)}$ is also a Poincaré map for $Y_{t}$,

- $q \in \operatorname{Sing}(Y) \cup P O\left(Y_{t}\right)$,

- $X_{t}(x)=Y_{t}(x)$ for $t \leq 0$.

Since $Y(y)=X(y)$ for $y \notin F_{X_{-1}(x)}\left(X_{t}, r_{0}, 1\right)$, we have

$$
W_{2 r_{0}}^{s}\left(p, X_{t}\right)=W_{2 r_{0}}^{s}\left(p, Y_{t}\right) \text { and } W_{2 r_{0}}^{u}\left(q, X_{t}\right)=W_{2 r_{0}}^{u}\left(q, Y_{t}\right) \text {. }
$$

As in the proof of proposition B in [15], by applying Lemma 4.2 for $Y$ and $f$, for any $\delta>0$ we can obtain $Z^{\delta} \in \mathcal{U}(X)$ and $r^{\prime}<r_{2}$ such that

- $Z^{\delta}$ is perturbation of $\varphi_{\delta} \in N_{\varepsilon(\delta)}\left(\Pi_{x, r_{0}}\right)$ satisfying $\varphi_{\delta}\left(V_{r^{\prime}}^{u}\left(x, Y_{t}\right)\right) \cap$ $V\left(x, Y_{t}\right)=\emptyset$,

- $Z^{\delta}(y)=Y(y)$ if $y \notin F_{x}\left(Y_{t}, r_{0}, 1\right)$,

- $g^{\prime}(y)=f \circ \varphi_{\delta}(y)$ if $y \in \Pi_{x, r_{0}}$,

- $d_{C^{1}}\left(Y, Z^{\delta}\right)<\delta$,

where $g^{\prime}: \Pi_{x, r_{0}} \longrightarrow \Pi_{Y_{1}(x)}$ is the Poincaré map induced by $Z_{t}^{\delta}$.

For the $C^{1}$ vector field $Y$ and $x \in W^{s}\left(p, Y_{t}\right) \cap W^{u}\left(q, Y_{t}\right)$, we have numbers $\varepsilon^{\star}>0$ and $\eta^{\star}$ satisfying Lemma 5.1. Choose a sufficiently small constant $\varepsilon>0$ with $\varepsilon<\min \left\{\frac{\varepsilon^{\star}}{2}, \frac{r^{\prime}}{2}, \frac{\eta^{\star}}{2}, \frac{\mu}{2}\right\}$, and let $0<\delta<\varepsilon$ be as in the definition of the orbital inverse shadowing of $Y_{t}$. So $Z^{\delta}$ constructed as the above is an element of $\mathcal{T}_{d}\left(\delta, Y_{t}\right)$. For simplicity, we denote $Z^{\delta}$ by $Z$. By the notion of the orbital inverse shadowing of $Y$, for the point $x$, there is a point $y \in M$ such that

$$
d_{H}\left(\overline{O\left(x, Y_{t}\right)}, \overline{O\left(y, Z_{t}\right)}\right)<\varepsilon .
$$

By applying Lemma 5.1, we can derive a contradiction as in the Case (1.1).

This completes the proof of Proposition B. 
Proof of Proposition C. Suppose that $\gamma, \gamma^{\prime} \in P O\left(X_{t}\right)$ are hyperbolic and $x \in$ $W^{s}\left(\gamma, X_{t}\right) \cap W^{u}\left(\gamma^{\prime}, X_{t}\right)$. Fix $p \in \gamma\left(X_{T}(p)=p\right)$, and let $r_{0}>0$ be sufficiently small so that we can define the Pioncaré map $f: \Pi_{p, r_{0}} \longrightarrow \Pi_{p}$. Since $p$ is hyperbolic, there are a $D f$-invariant splitting $\hat{\Pi}_{p}=E_{p}^{s} \oplus E_{p}^{u}$ and two constants $C>0,0<\lambda<1$ such that $\left\|D f_{\mid E_{p}^{s}}^{m}\right\|<C \lambda^{m}$ and $\left\|D f_{\mid E_{p}^{u}}^{-m}\right\|<C \lambda^{m}$ for all $m \geq$

0 . Let $W_{r}^{\sigma}(p, f)$ be the connected component of $W^{\sigma}\left(\gamma, X_{t}\right) \cap \Pi_{p, r}$ containing $p$ for $\sigma=s, u$ and $0<r \leq r_{0}$. Suppose that $x \in W_{r_{0} / 2}^{s}(p, f) \backslash \operatorname{int} W_{r_{0} / 2}^{s}(p, f)$, and let $T^{\prime}>0$ be the number with $f(x)=X_{T^{\prime}}(x)$ and take $0<r_{1}<\frac{r_{0}}{4}$ such that $F_{p}\left(X_{t}, r_{1}, T\right) \cap F_{x}\left(X_{t}, r_{1}, T^{\prime}\right)=\emptyset$.

Before continuing our proof, we will cite the following result from [15].

Lemma 5.2 ([15, Lemma 4.1]). Under the above notation, for every $C^{1}$ neighborhood $\mathcal{U}(X)$ of $X$, there are $0<\varepsilon_{0}<\frac{r_{0}}{4}$ and $Y \in \mathcal{U}(X)$ satisfying

(i) $Y(y)=X(y)$ if $y \notin F_{p}\left(X_{t}, r_{1}, T\right) \cup F_{x}\left(X_{t}, r_{1}, T^{\prime}\right)$,

(ii) $\gamma, \gamma^{\prime} \in P O\left(Y_{t}\right)$ and $Y_{T}(p)=p \in \gamma$,

(iii)

$$
g(y)= \begin{cases}\exp _{p} \circ D_{p} f \circ \exp _{p}^{-1}(y) & \text { if } y \in B_{\varepsilon_{0} / 4}(p) \cap \Pi_{p, r_{0}} \\ f(y) & \text { if } y \notin B_{\varepsilon_{0}}(p) \cap \Pi_{p, r_{0}},\end{cases}
$$

(iv) $g(p)=p, x \in W_{r_{0}}^{s}(p, g)$ and $T_{x} W_{r_{0}}^{s}(p, g)=T_{x} W_{r_{0}}^{s}(p, f)$,

(v) $T_{x} W^{u}\left(\gamma^{\prime}, Y_{t}\right)=T_{x} W^{u}\left(\gamma^{\prime}, X_{t}\right)$.

Here $g: \Pi_{p, r_{0}} \longrightarrow \Pi_{p}$ is the Poincaré map of $Y_{t}$ and $W_{r_{0}}^{\sigma}(p, g)$ is the connected component of $W^{\sigma}\left(\gamma, Y_{t}\right) \cap \Pi_{p, r_{0}}$ containing $p(\sigma=s, u)$.

Put $E_{x}^{\sigma}(\varepsilon)=\left\{v \in E_{x}^{\sigma} \mid\|v\| \leq \varepsilon\right\}$ for $\varepsilon>0(\sigma=s, u)$, and $g \in \operatorname{Emb}^{1}\left(\Pi_{p, r_{0}}, \Pi_{p}\right)$, $p=g(p) \in \Pi_{p}$ and $\varepsilon_{0}>0$ be given by Lemma 5.2. Then $\exp _{p}\left(E_{p}^{\sigma}\left(\frac{\varepsilon_{0}}{4}\right)\right) \subset$ $W_{r_{0}}^{\sigma}(p, g)$ and $\operatorname{dim} \exp _{p}\left(E_{p}^{\sigma}\left(\frac{\varepsilon_{0}}{4}\right)\right)=\operatorname{dim} W_{r_{0}}^{\sigma}(p, g)$ for $\sigma=s, u$ since $\varepsilon_{0}$ is small. For convenience, we denote $\exp \left(E_{p}^{\sigma}(\varepsilon)\right)$ by $W_{\varepsilon}^{\sigma}(p, g)$ for $\sigma=s, u$ and $0<\varepsilon \leq \frac{\varepsilon_{0}}{4}$.

Let $X \in O I S^{\circ}(M)$, suppose that $\gamma, \gamma^{\prime} \in P O\left(X_{t}\right)$ are hyperbolic and $x \in$ $W^{s}\left(\gamma, X_{t}\right) \cap W^{u}\left(\gamma^{\prime}, X_{t}\right)$. Let $p \in \gamma\left(X_{T}(p)=p, T>0\right)$ and $f: \Pi_{p, r_{0}} \longrightarrow \Pi_{p}$ $\left(r_{0}>0\right)$ be as before. We may assume that

- $x \in W_{r_{0} / 2}^{s}(p, f) \backslash \operatorname{int} W_{r_{0} / 2}^{s}(p, f)$,

- $W_{2 r_{0}}^{u}\left(\gamma^{\prime}, X_{t}\right) \cap \Pi_{p, r_{0}}$.

Here, $W_{r_{0}}^{u}\left(\gamma^{\prime}, X_{t}\right)$ is the local unstable manifold of $\gamma^{\prime}$. Fix a $C^{1}$ neighborhood $\mathcal{U}(X) \subset O I S^{\circ}(M)$ of $X$, and let $0<\varepsilon_{0}<\frac{r_{0}}{4}, Y \in \mathcal{U}(X)$ and $g$ be given by Lemma 5.2. Thus $T_{x} W_{r_{0}}^{s}(p, g)=T_{x} W_{r_{0}}^{s}(p, f), W_{2 r_{0}}^{u}\left(\gamma^{\prime}, X_{t}\right)=W_{2 r_{0}}^{u}\left(\gamma^{\prime}, Y_{t}\right)$, and $X_{t}(x)=Y_{t}(x)$ for all $t \leq 0$. Clearly, $1 \leq \operatorname{dim} W_{r_{0}}^{s}(p, g) \leq \operatorname{dim} \Pi_{p}$ and $1 \leq$ $\operatorname{dim}\left(W_{r_{0}}^{u}\left(\gamma^{\prime}, Y_{t}\right) \cap \Pi_{p}\right) \leq \operatorname{dim} \Pi_{p}$. If $\operatorname{dim} W_{r_{0}}^{s}(p, g)=\operatorname{dim} \Pi_{p}$ or $\operatorname{dim}\left(W_{r_{0}}^{u}\left(\gamma^{\prime}, Y_{t}\right) \cap\right.$ $\Pi_{p}=\operatorname{dim} \Pi_{p}$, then the conclusion is clear.

Pick $l>0$ so large that $g^{l-1}(x) \in W_{\varepsilon_{0} / 8}^{s}(p, g)$ and let $C^{u}\left(g^{l}(x)\right)$ be the connected component of $W^{u}\left(\gamma^{\prime}, Y_{t}\right) \cap \Pi_{p}$ containing $g^{l}(x)$. To simplify the notation, denote $g^{l}(x)$ by $x$. Then we get

$$
\exp _{p}^{-1}\left(C^{u}(x)\right) \subset \hat{\Pi}_{p} \text { and } T_{x} C^{u}(x)=T_{x}\left(W^{u}\left(\gamma^{\prime}, Y_{t}\right) \cap \Pi_{p}\right) .
$$


For a linear subspace $E$ of $\hat{\Pi}_{p}$ and $\nu>0$, let

$$
E_{\nu}(x)=\left\{v+\exp _{p}^{-1}(x) \mid v \in E \text { with }\|v\| \leq \nu\right\}
$$

be a piece of an affine space running parallel to $E$. Let $T^{\prime \prime}, T^{\prime \prime \prime}>0$ be numbers with $Y_{T^{\prime \prime}}\left(g^{-1}(x)\right)=x$ and $Y_{T^{\prime \prime \prime}}(x)=g(x)$, respectively. Choose a linear subspace $E^{\prime} \subset \hat{\Pi}_{p}$ and $0<\nu_{0}<\varepsilon_{0} / 8$ such that

- for every $0<\nu \leq \nu_{0}$, $\exp _{p}\left(E_{\nu}^{\prime}(x)\right) \subset B_{\varepsilon_{0} / 4}(p)$,

- $T_{x} \exp _{p}\left(E_{\nu_{0}}^{\prime}(x)\right)=T_{x} C^{u}(x)$,

- $\left(F_{g^{-1}(x)}\left(Y_{t}, \nu_{0}, T^{\prime \prime}\right) \cup F_{x}\left(Y_{t}, \nu_{0}, T^{\prime \prime \prime}\right)\right) \cap \gamma=\emptyset$,

- $\left\{Y_{t}\left(g^{-1}(x)\right): t<0\right\} \cap F_{g^{-1}(x)}\left(Y_{t}, \nu_{0}, T^{\prime \prime}\right)=\emptyset$ and $\left\{Y_{t}(x): t<0\right\} \cap F_{x}\left(Y_{t}, \nu_{0}, T^{\prime \prime \prime}\right)=\emptyset$,

- $B_{\nu_{0}}\left(\left\{Y_{t}(x): t \leq 0\right\}\right) \cap F_{x}\left(Y_{t}, \nu_{0}, T^{\prime \prime \prime}\right) \cap \Pi_{p, r_{0}}$ $=B_{\nu_{0}}(x) \cap F_{x}\left(Y_{t}, \nu_{0}, T^{\prime \prime \prime}\right) \cap \Pi_{p, r_{o}}$,

- $g^{i}\left(W_{r_{0}}^{s}(p, g) \cap B_{\nu_{0}}\left(g^{-1}(x)\right)\right) \cap B_{\nu_{0}}\left(g^{-1}(x)\right)=\emptyset \quad$ for $i \geq 1$,

- $Y_{-t}\left(C^{u}\left(g^{-1}(x)\right)\right) \cap B_{\nu_{0}}\left(g^{-1}(x)\right)=\emptyset \quad$ for all $t>0$.

Here $C^{u}\left(g^{-1}(x)\right)$ is the connected component of $W^{u}\left(\gamma^{\prime}, Y_{t}\right) \cap \Pi_{p} \cap B_{\nu_{0}}\left(g^{-1}(x)\right)$ containing $g^{-1}(x)$.

Lemma 5.3 ([14, Lemma 4.2]). Fix a $C^{1}$ neighborhood $\mathcal{U}(Y) \subset \mathcal{U}(X)$ of $Y$. Then there are $0<\nu_{1}<\frac{\nu_{0}}{4}$ and $Y^{\prime} \in \mathcal{U}(Y)$ such that

(i) $Y^{\prime}(y)=Y(y)$ if $y \notin F_{g^{-1}(x)}\left(Y_{t}, \nu_{0}, T^{\prime \prime \prime}\right)$,

(ii) $Y_{T^{\prime \prime}}^{\prime}\left(g^{-1}(x)\right)=x$,

(iii) $\exp _{p}\left(E_{\nu_{1}}^{\prime}(x)\right) \subset W^{u}\left(\gamma^{\prime}, Y_{t}^{\prime}\right) \cap \Pi_{p}$ and $T_{x} \exp _{p}\left(E_{\nu_{1}}^{\prime}(x)\right)=T_{x}\left(W^{u}\left(\gamma^{\prime}, Y_{t}^{\prime}\right) \cap \Pi_{p}\right)$.

Remark 5.4. (i) We see that $W_{2 r_{0}}^{u}\left(\gamma^{\prime}, Y_{t}\right)=W_{2 r_{0}}^{u}\left(\gamma^{\prime}, Y_{t}^{\prime}\right)$ and $Y_{t}(x)=$ $Y_{t}^{\prime}(x)$ for $t \leq 0$.

(ii) Let $g^{\prime}: \Pi_{p, r_{0}} \longrightarrow \Pi_{p}$ be the Pioncaré map induced by $Y_{t}^{\prime}$. Then by Lemma 5.3(i), $\gamma^{\prime} \in P O\left(X_{t}\right)$ and $g^{\prime}(y)=g(y)$, if $y \in \Pi_{p, r_{0}} \backslash B_{\nu_{0}}\left(g^{-1}(x)\right)$. Thus $g^{\prime}(p)=g(p)$. By Lemma 5.3(ii), $g^{\prime i}(x)=g(x)$ for all $i \geq 0$.

(iii) By the perturbation used in the above lemma, $W_{\varepsilon_{0} / 4}^{s}(p, g)$ may be deformed near $g^{-j}(x) \in W_{\varepsilon_{0} / 4}^{s}(p, g)$ for some $j>0$. We denote the deformed manifold by $W_{\varepsilon_{0} / 4}^{s}\left(p, g^{\prime}\right)$. Then $x \in W_{\varepsilon_{0} / 4}^{s}\left(p, g^{\prime}\right)$ and $T_{x} W_{\varepsilon_{0} / 4}^{s}\left(p, g^{\prime}\right)=T_{x} W_{\varepsilon_{0} / 4}^{s}(p, g)$.

If $\exp _{p}\left(E_{\nu_{1}}^{\prime}(x)\right)$ does not meet $W_{\varepsilon_{0} / 4}^{s}\left(p, g^{\prime}\right)$ transverse at $x$, then the piece $E_{\nu}^{\prime}(x)$ of the affine space is not transversal with respect to the local linear stable manifold $E_{p}^{s}\left(\frac{\varepsilon_{0}}{4}\right)$ of $p$. Thus we can destroy the intersection "locally" with a $C^{1}$ small perturbation (for more details, see [15]).

To simplify the notation, denote $Y^{\prime}, g^{\prime}$ and $W_{\varepsilon_{0} / 4}^{s}\left(p, g^{\prime}\right)$ by $Y, g$, and $W_{\varepsilon_{0} / 4}^{s}(p, g)$, respectively. If $\exp _{p}\left(E_{\nu_{1}}^{\prime}(x)\right)$ dose not meet $W_{\varepsilon / 4}^{s}(p)$ transversely at $x$, then there is $0<r^{\prime}<\frac{\nu_{1}}{2}$ such that 
- for every $\delta>0$, there is $\psi_{\delta} \in N_{\varepsilon(\delta)}\left(\Pi_{x, 2 \nu_{1}}\right)$ satisfying

$$
\left\{\begin{array}{l}
\psi_{\delta}\left(\exp _{p}\left(E_{\nu_{1}}^{\prime}(x)\right) \cap B_{r^{\prime}}(x)\right) \cap W_{r_{0}}^{s}(p)=\emptyset, \\
\psi_{\delta}(y)=y \text { if } y \notin \Pi_{x, \nu_{1}},
\end{array}\right.
$$

- $\exp _{p}\left(E_{\nu_{1}}^{\prime}(x)\right) \cap B_{r^{\prime}}(x)=\left[\bigcup_{0 \leq t \leq t_{2}} Y_{t}\left(W_{2 r_{0}}^{u}\left(\gamma^{\prime}, Y_{t}\right)\right)\right] \cap \Pi_{p, r_{0}} \cap B_{r^{\prime}}(x)$,

where $Y_{-t_{2}}(x) \in W_{r_{0}}^{u}\left(\gamma^{\prime}, Y_{t}\right)$.

For the $C^{1}$ vector field $Y$ and $x \in W^{s}\left(\gamma, Y_{t}\right) \cap W^{u}\left(\gamma^{\prime}, Y_{t}\right)$, we have numbers $\varepsilon^{\star}>0$ and $\eta^{\star}>0$ satisfying the conclusion of Lemma 5.1. Choose a sufficiently small constant $\varepsilon>0$ with $\varepsilon<\min \left\{\frac{\varepsilon^{\star}}{2}, \frac{r^{\prime}}{2}, \frac{\eta^{\star}}{2}\right\}$, and let $0<\delta<\varepsilon$ be the corresponding number with respect to $\varepsilon$ in the definition of the orbital inverse shadowing of $Y$. By Lemma 4.2, we can construct $Z \in \mathcal{U}(Y)$ for the above perturbation such that

$$
\begin{cases}Z(z)=Y(z) & \text { if } \quad z \notin F_{x}\left(Y_{t}, 2 \nu_{1}, T^{\prime \prime \prime}\right), \\ \tilde{g}(z)=g \circ \psi_{\delta}(z) & \text { if } \quad z \in \Pi_{x, 2 \nu_{1}}, \\ d_{C^{1}}(Y, Z)<\delta . & \end{cases}
$$

Here $\tilde{g}: \Pi_{x, 2 \nu_{1}} \longrightarrow \Pi_{g(x)}$ is the Poincaré map induced by $Z_{t}$. Because $Z$ is in $\mathcal{T}_{d}(\delta, 1, Y)$, there is $y \in M$ such that

$$
d_{H}\left(\overline{O\left(x, Y_{t}\right)}, \overline{O\left(y, Z_{t}\right)}<\varepsilon .\right.
$$

Because $Z(y)=Y(y)$ for $y \notin F_{x}\left(Y_{t}, 2 \nu_{1}, T^{\prime \prime \prime}\right)$,

$$
W_{2 r_{0}}^{s}\left(\gamma, Z_{t}\right)=W_{2 r_{0}}^{s}\left(\gamma, Y_{t}\right) \text { and } W_{2 r_{0}}^{u}\left(\gamma, Z_{t}\right)=W_{2 r_{0}}^{u}\left(\gamma, Y_{t}\right)
$$

Similar to the proof of Proposition B, we can get a contradiction. This completes the proof of Proposition C.

Acknowledgements. The authors wishes to express their appreciation to the referee for his careful reading of the manuscript and valuable suggestions.

\section{References}

[1] T. Choi, K. Lee, and M. Lee, Inverse shadowing for suspension flows, preprint.

[2] R. Corless and S. Pilyugin, Approximate and real trajectories for generic dynamical systems, J. Math. Anal. Appl. 189 (1995), no. 2, 409-423.

[3] P. Diamond, K. Lee, and Y. Han, Bishadowing and hyperbolicity, Internat. J. Bifur. Chaos Appl. Sci. Engrg. 12 (2002), no. 8, 1779-1788.

[4] S. Gan and L. Wen, Nonsingular star flows satisfy Axiom A and the no-cycle condition, Invent. Math. 164 (2006), no. 2, 279-315.

[5] Y. Han and K. Lee, Inverse shadowing for structurally stable flows, Dyn. Syst. 19 (2004), no. 4, 371-388.

[6] S. Hayashi, On the solution of $C^{1}$ stability conjecture for flows, Ann. Math. 353 (1997), 3391-3408.

[7] P. Kloeden and J. Ombach, Hyperbolic homeomorphisms and bishadowing, Ann. Polon. Math. 65 (1997), no. 2, 171-177.

[8] P. Kloeden, J. Ombach, and A. Pokrovskii, Continuous and inverse shadowing, Funct. Differ. Equ. 6 (1999), no. 1-2, 137-153.

[9] K. Lee, Continuous inverse shadowing and hyperbolicity, Bull. Austral. Math. Soc. 67 (2003), no. 1, 15-26. 
[10] K. Lee and Z. Lee, Inverse shadowing for expansive flows, Bull. Korean Math. Soc. 40 (2003), no. 4, 703-713.

[11] K. Lee and J. Park, Inverse shadowing of circle maps., Bull. Austral. Math. Soc. 69 (2004), no. 3, 353-359.

[12] K. Lee and K. Sakai, Various shadowing properties and their equivalence, Discrete Contin. Dyn. Syst. 13 (2005), no. 2, 533-540.

[13] _ Structural stability of vector fields with shadowing, J. Differential Equations 232 (2007), no. 1, 303-313.

[14] R. Mañé, A proof of the $C^{1}$ stability conjecture, Inst. Hautes Etudes Sci. Publ. Math. No. 66 (1988), 161-210.

[15] K. Moriyasu, K. Sakai, and N. Sumi, Vector fields with topological stability, Trans. Amer. Math. Soc. 353 (2001), no. 8, 3391-3408.

[16] K. Moriyasu, K. Sakai, and W. Sun, $C^{1}$ stably expansive flows, J. Differential Equations 213 (2005), no. 2, 352-367.

[17] S. Pilyugin, Shadowing in structurally stable flows, J. Differential Equations 140 (1997), no. 2, 238-265.

[18] - Inverse shadowing by continuous methods, Discrete Contin. Dyn. Syst. 8 (2002), no. 1, 29-38.

[19] S. Pilyugin, A. Rodionova, and K. Sakai, Orbital and weak shadowing properties, Discrete Contin. Dyn. Syst. 9 (2003), no. 2, 287-308.

[20] O. Plamenevskaya, Pseudo-orbit tracing property and limit shadowing property on a circle, Vestnik St. Petersburg Univ. Math. 30 (1997), no. 1, 27-30.

[21] C. Robinson, Structural stability of vector fields, Ann. of Math. (2) 99 (1974), 154-175.

[22] _ Stability theorems and hyperbolicity in dynamical systems, Rocky Mountain J. Math. 7 (1977), no. 3, 425-437.

[23] K. Sakai, Pseudo orbit tracing property and strong transversality of diffeomorphisms on closed manifolds, Osaka J. Math. 31 (1994), no. 2, 373-386.

[24] R. Thomas, Topological stability: some fundamental properties, J. Differential Equations 59 (1985), no. 1, 103-122.

[25] L. Wen, On the $C^{1}$ stability conjecture for flows, J. Differential Equations 129 (1996), no. 2, 334-357.

[26] K. Yano, Topologically stable homeomorphisms of the circle, Nagoya Math. J. 79 (1980), $145-149$.

KeOnheE LeE

Department of Mathematics

Chungnam National University

DAEJEOn 305-764, Korea

E-mail address: khlee@math.cnu.ac.kr

ZOONHEE LEE

Department of Mathematics

Chungnam National University

DAEJEON 305-764, KoreA

YONG ZHANG

Department of Mathematics

SuZHOU UNIVERSITY

Suzhou, China

E-mail address: yongzhang@suda.edu.cn 